\section{Cahiers de Narratologie}

Analyse et théorie narratives

$10.2 \mid 2001$

La voix narrative

\title{
L'écriture polyphonique dans La pharisienne de François Mauriac
}

\section{Alain Tassel}

\section{(2) OpenEdition}

1 Journals

Édition électronique

URL : http://journals.openedition.org/narratologie/10301

DOI : 10.4000/narratologie.10301

ISSN : 1765-307X

Éditeur

LIRCES

\section{Édition imprimée}

Date de publication : 1 janvier 2001

Pagination : 463-475

ISBN : 2914561032

ISSN : 0993-8516

\section{Référence électronique}

Alain Tassel, «L'écriture polyphonique dans La pharisienne de François Mauriac », Cahiers de Narratologie [En ligne], 10.2 | 2001, mis en ligne le 01 janvier 2001, consulté le 11 juin 2020. URL:

http://journals.openedition.org/narratologie/10301;DOI : https://doi.org/10.4000/narratologie.10301 


\title{
L'ÉCRITURE POLYPHONIQUE DANS LA PHARISIENNE DE FRANÇOIS MAURIAC
}

\author{
Alain TASSEL \\ Université de Nice-Sophia Antipolis
}

Ecrivant La Pharisienne « avec une hâte fiévreuse ${ }^{1}$ » au cours du second semestre de l'année 1940, Mauriac renoue avec la littérature de combat en abordant des questions qui, le passionnent depuis sa jeunesse et ont essaimé dans toute son oeuvre. Il prend une nouvelle fois pour cible la figure de la pharisienne, dans laquelle il reconnaît lui-même « un personnage central [de son] œuvre [...] qui a été toujours présent sous une forme ou sous une autre ${ }^{2} »$. Ce roman est ainsi porté par une visée critique clairement explicitée dans un entretien avec Jean Amrouche : «J'ai su très précisément contre quoi je m'élevais et ce que je voulais dénoncer [...] j'ai vraiment centré ce livre autour de la folle dévotion ${ }^{3} »$. Le romancier exerce sa verve contre la fausse piété, contre la religion des apparences et des certitudes, une religion dévoyée qui sert d'instrument à la volonté de puissance. Cette disposition plonge des racines troubles et lointaines dans sa propre vie, ce qu'il admet lors de ce même entretien, où il ajoute : " Il y a dans beaucoup de mes personnages, dans presque tous mes héros, une volonté de puissance forcenée ${ }^{4}$ ». Dans $L a$ Pharisienne, non seulement le romancier dresse le bilan amer des confusions du pharisaïsme et de la foi, mais il explore les sources et les manifestations de la volonté de puissance, une

${ }^{1}$ François Mauriac, Paroles perdues et retrouvées, Paris, Grasset, 1986, p. 60.

${ }^{2}$ François Mauriac, Souvenirs retrouvés, Paris, Fayard/INA, 1981, p. 257.

\footnotetext{
${ }^{3}$ Ibid., p. 247.

${ }^{4}$ Ibid., p. 254.
} 
énergie mobilisée pour dominer les êtres sous la forme, notamment, de la direction de conscience.

Mais ce n'est pas seulement à cause du nouveau regard, encore plus acerbe, porté sur ces préoccupations récurrentes que Mauriac considérait La Pharisienne comme « un de [ses] livres les meilleurs, un de ceux où l'expérience religieuse aura en [lui] le mieux servi le romancier ${ }^{5} »$. De fait, ce roman marque une étape importante dans la carrière littéraire de Mauriac, en raison du remaniement significatif apporté à sa technique narrative, à la suite des critiques de Sartre publiées dans la $N R F$, en février 1939. Certes, les exclusives sartriennes s'éclairent à la lumière d'options anthropologiques et esthétiques défendues avec l'âpreté et la virulence d'un manifeste littéraire. Mais, en dépit de ses déclarations, Mauriac a été affecté par les reproches portés sur l'absence de liberté de ses personnages, sur les interventions d'auteur supposées nuire à l'intérêt de la lecture : "Sartre a éreinté $L a$ Fin de la nuit dans un article assez retentissant [...] Vous savez, ça ne m'a pas du tout désespéré [...] Personnellement ça m'a fait réfléchir sur mon métier ${ }^{6} »$. Cet article l'a donc conduit à s'interroger sur les relations du narrateur et des personnages, à mettre au point une technique narrative propre à rendre sensible la présence de l'autre, à accorder aux principaux protagonistes une existence autonome. Persuadé de la fécondité de cette nouvelle orientation esthétique, Mauriac s'est plu à ironiser sur les heureuses et imprévisibles incidences de l'éreintement sartrien: "Je l'ai dit pour taquiner Sartre, mais c'est un peu vrai : je crois que si j'ai eu le prix Nobel, c'est un peu grâce à lui. En effet, à partir de là, j'ai travaillé La Pharisienne d'une certaine manière ; enfin j'ai eu un second départ ${ }^{7} »$. Dans quelle mesure cette nouvelle manière trouve-t-elle sa source dans l'orchestration des voix, dans le réglage de leur croisement ? Comment Mauriac tentet-il de se libérer d'une perspective dominante et d'un discours

${ }^{5}$ François Mauriac, Euvres romanesques et théâtrales complètes, Paris, Gallimard, coll. La Pléiade, édition de Jacques Petit, tome 3, p. 926. (sigle: III).

${ }^{6}$ François Mauriac, Les Paroles restent, Paris, Grasset, 1985, p. 85.

${ }^{7}$ Ibid., p. 85. 
centralisateur pour laisser sourdre les vibrations et les éclats de ces voix contradictoires?

L'effet de stéréophonie, de relief acoustique est obtenu par la singularisation des discours ou des pensées des personnages, par l'inscription d'une autonomie discursive.

Sensible aux reproches de Sartre, Mauriac crée un personnage de narrateur-témoin, qui observe de l'extérieur les agissements de Brigitte Pian, assiste à des scènes mettant aux prises Jean de Mirbel et Michelle. A la faveur d'un jeu de miroirs, les objections sartriennes se trouvent incorporées au texte sous la forme d'un pastiche : "Quelqu'un me demande : "Comment connaissez-vous tous ces événements auxquels vous n'avez pas assisté ? De quel droit reproduisez-vous des conversations que vous n'avez pas entendues ?"8 ». En plus de sa portée ludique, le pastiche est investi d'une fonction narrative : il motive la divulgation de l'origine des informations, des sources orales et écrites. Le récit de Louis Pian est reconstitué à partir des témoignages de son instituteur, M. Puybaraud, et de sa sœur, Michelle, des carnets et des notes de l'abbé Calou, des lettres de Mirbel. Si le narrateur s'arroge « [le] droit d'ordonner cette matière, d'orchestrer ce rée $1^{9}$ ", il incorpore à sa parole cette hétérogénéité énonciative, s'emploie à singulariser les voix des personnages mis en scène.

Ainsi, les propos ou les pensées attribués au jeune Louis Pian ou à Jean de Mirbel portent-ils l'empreinte d'un idiolecte de l'enfance, que révèle tantôt une redondance syntaxique insérée dans un dialogue, - «ma mère à moi ${ }^{10} »-$, tantôt un terme familier lesté d'une connotation dépréciative, - « Ils me traitent comme un gosse $11 »-$, tantôt une formulation enfantine caractérisant les réflexions d'un narrateur-personnage désorienté par les projets contradictoires de ses parents:

\footnotetext{
8 III, p. 734.

9 III, p. 735.

10 III, p. 712.

11 III, p. 739.
} 
"Que les grandes personnes s'arrangent entre elles! 12 ». Le marquage typographique, en l'occurrence l'emploi de l'italique, désigne une tournure étrangère au discours du narrateur, référant, par exemple, aux tabous sexuels de l'enfance : "Peut-être, en ce moment [Jean] racontait-il à Michelle ce que j'appelais des histoires de saletés ${ }^{13} »$. De même, l'acception idiolectale de l'adjectif « instruit », utilisé absolument par Jean de Mirbel pour référer à un savoir relatif à la vie sexuelle, est signalée par l'italique tout en étant assortie d'un commentaire métatextuel, une glose justifiant la différence typographique : "Cette fois je compris : "Croyezvous qu'il soit instruit ?" demanda-t-il.

"Etre instruit", dans notre langue, cela signifiait connaître les secrets de la vie, les mystères de la procréation ${ }^{14} »$.

Dans ce roman de l'adolescence, c'est le personnage de Jean de Mirbel qui est individualisé le plus efficacement, par le biais d'une large palette de procédés qui concourent à un effet d'oralité. La vivacité de son expression verbale est connotée par l'emploi de l'exclamatif, du pronom démonstratif «ça » ou, plus fréquemment encore, de locutions propres à la langue orale, telle cette amitié entre Mirbel et Louis Pian nouée " à la vie, à la mort ${ }^{15}$ ». Les paroles de Mirbel retentissent d'accents de bravade et de cynisme, rendus sensibles par la présence de locutions familières au sein du discours indirect libre : «tous les samedis, confession : il avouait n'importe quoi...tous les dimanches, communion: qu'est-ce que ça pouvait faire, de ne pas y croire ? Ca ne faisait ni chaud ni froid ${ }^{16} »$. La scène de voyeurisme du chapitre VIII, où, à la faveur d'une restriction de champ, Jean surprend sa mère et son amant, Raoul, dans une variante de scène primitive, illustre la porosité du discours indirect libre aux traits de l'oralité : «Mais non, ils se lèveraient tard, ils avaient tout leur

\footnotetext{
12 III, p. 713.

13 III, p. 740.

${ }^{14} \mathrm{Ibid}$.

${ }^{15}$ III, p. 748.

16 III, p. 747.
} 
temps, les salauds... Quels salauds, tout de même... Non pas de faire l'amour, mais de faire les mijaurées... Lui, il avait fini de marcher... Il ne marcherait jamais plus, bien sûr, et pour personne. Tout le monde couche avec tout le monde : c'est ça la vie ${ }^{17} »$.

L'immixtion des modalités spécifiques du discours au cœur du récit de pensée donne tout leur relief aux réflexions gouailleuses et désabusées de Jean, leur confère les vibrations d'une voix travaillée par la révolte et le désespoir. Le romancier use avec beaucoup d'habileté des décrochages énonciatifs dans cet épisode. Le croisement des voix tire ses effets du changement de modalité narrative : «Et tout à coup, une panique le prit à l'idée d'être découvert, d'avoir à lui parler, à entendre ses explications. Quelle horreur, ce visage imaginé de sa mère, honteuse et suppliante ! 18 ». L'analyse psychologique assumée par le narrateur s'organise autour de la pulsion de fuite qui s'empare de l'adolescent, alors que l'irruption soudaine du discours indirect libre laisse sourdre sa souffrance. La désacralisation de la mère, engluée dans le mensonge, préfigure le glissement vers la débauche et le mal de cet ange noir. Ainsi, en vertu d'un phénomène spéculaire très éprouvé, la relation du lecteur au texte redouble-t-elle celle qu'entretient le personnage focal avec les amants. Le lecteur a accès à l'intériorité de l'adolescent qui surprend sa mère et son amant dans leur étreinte.

L'hétérogénéité énonciative est également mise à contribution pour singulariser l'emprise verbale qu'exerce Brigitte Pian sur son entourage. La modalisation autonymique $^{19}$ en discours second (l'usage et la mention d'une forme discursive propre à Brigitte Pian) est obtenue par

$$
\begin{aligned}
& 17 \text { III, p. } 785 . \\
& 18 \text { III, p. } 784 .
\end{aligned}
$$

${ }^{19}$ Sur cette notion, on se référera avec profit à deux contributions de Jacqueline Authier-Revuz.

1 - « Hétérogénéité montrée et hétérogénéité constitutive : éléments pour une approche de l'autre dans le discours », DRLAV, $\mathrm{n}^{\circ} 26,1982$, pp. $91-151$

2 - « Repères dans le champ du discours rapporté ", L'information grammaticale, $\mathrm{n}^{\circ} 54$, juin 1992, pp. 38-42, et $\mathrm{n}^{\circ} 56$, janvier 1993, pp. $10-15$ 
l'emploi des guillemets : " ma belle-mère trouvait [l'abbé Calou] de jugement faible et bizarre et comme elle disait "l'avait à l'œil" 20 ». Ailleurs, la modalisation autonymique en discours indirect affecte un îlot textuel plus étendu : « Brigitte murmura d'une voix égarée "que ces enfants la rendraient folle"21 ». Si les guillemets signalent le changement de voix, la polyphonie, ils entretiennent surtout l'illusion d'une restitution littérale des propos. Il arrive que les guillemets, très fréquemment employés dans ce roman ( soixante occurrences, alors qu'on en dénombre 27 dans Le Sagouin ${ }^{22}$ ), introduisent aussi bien un discours qu'un commentaire du narrateur. Référons-nous, par exemple à l'une des antonomases désignant Brigitte Pian : "Il est vrai que j'étais le petit Pian, le beau-fils de Mme Brigitte "la bienfaitrice"23 ». Le contexte nous permet d'attribuer l'appellatif "Mme Brigitte »-utilisé sans guillemets -, à Jean de Mirbel, tandis que l'usage des guillemets pour introduire l'antonomase soulève une ambiguïté. Les guillemets peuvent révéler la présence d'un discours second, signaler une intonation ironique escortant la prononciation de ce mot par Jean de Mirbel, ou encore marquer une distance énonciative, d'ordre antiphrastique, de la part du narrateur.

L'efficacité de ce dialogisme romanesque tient à ce que les personnages sont saisis dans des contextes discursifs très contrastés où se manifestent les diverses facettes de leur univers de parole. Souvent, le discours second s'introduit dans la langue du narrateur, puis se développe à son détriment en faisant ressortir les marques de la subjectivité du personnage. Ainsi en est-il des propos que la Pharisienne tient sur ses élèves en présence de Louis Pian : "elle devait, à onze heures, faire le catéchisme aux enfants de la Première

${ }^{20}$ III, p. 769.

21 III, p. 836.

22 Voir Jean-Claude Bibolet, "L'utilisation stylistique des guillemets », dans Lectures du Sagouin, Reims, Presses Universitaires, 1987, pp. 85-89.

${ }^{23}$ III, p. 714 
Communion qui, à l'entendre, étaient sournois et stupides, incapables de rien comprendre à rien et ne se plaisant qu'à se pincer naturellement dans leurs parties charnues. Sales, avec cela, souillant les parquets et sentant mauvais. Quant au moindre sentiment de gratitude, ah! bien ouiche ! on pouvait s'éreinter à leur service : les parents seraient les premiers, le cas échéant, à piller votre maison et à vous assassiner ${ }^{24} »$. Le discours indirect libre crée une étonnante impression de proximité avec le personnage, répercute quelques échos de son idiome : la tournure familière «rien comprendre à rien » ressortit au sarcasme dont Brigitte Pian enveloppe les êtres vulnérables qu'elle méprise. Chez elle, la haine se masque sous le discours de la charité.

Mauriac sollicite habilement toute la gamme du discours rapporté : le texte se nuance des inflexions de cette voix féminine ou retentit de ses éclats. Dans la scène théâtrale relatant son affrontement avec Michèle de Mirbel, les vibrations de sa voix trahissent le glissement du soutien absolu à l'insinuation perfide : " Je veux croire, je crois de toute mon âme à son innocence, à sa relative innocence ${ }^{25} »$. Ces nuances, ces retours et replis façonnent le discours reptilien de Brigitte Pian, dont la calomnie est assourdie, à demi-étouffée par le style indirect libre: "Mais les filles-mères de quatorze et quinze ans, ça existait. On voyait bien qu'il ne visitait pas les pauvres, lui !26». Une modulation plus brutale, plus abrupte est opérée avec le passage au discours direct. Le cri « Toutes des chiennes ", proféré après l'annonce de la liaison de Puybaraud et d'Octavie, est relayé par l'accusation, mêlée de répulsion, lancée contre sa belle-fille : "Michèle est une fille qui aime l'homme ${ }^{27}$ ». Ces accents de puritanisme révèlent moins un dégoût de la sensualité qu'ils ne dissimulent une frustration née du refoulement du désir sexuel, une convoitise mal réfrénée. En outre, la tentation de l'angélisme, qui se profile à l'arrière-plan de ces propos, n'a pas épargné le

24 III, p. 788.

25 III, p. 790.

26 Ibid.

27 III, p. 789. 
romancier comme l'indique une variante consignée dans la notice de La Pléiade : "le malheur des hommes et le mien en particulier, c'est de n'être pas chastes ${ }^{28}$ ».

Enfin, en raison d'un degré mimétique plus élevé que celui de l'analyse intellectuelle, le monologue narrativisé favorise l'émergence de la manie logicienne de Brigitte Pian, tout en soumettant les soubresauts, les sinuosités de cette pensée à l'effet de lissage propre au récit. Les réflexions que lui inspire l'amour de Michèle pour Jean de Mirbel portent l'empreinte d'une casuistique : «Oui, selon l'esprit du monde, elle aurait dû tirer avantage de ce scandale ; mais non, elle le ferait tourner au salut de cette misérable enfant. Que cette petite eût, sinon touché le fond de l'abîme, du moins qu'elle l'eût si jeune approché, c'était un malheur certes, mais qui rendrait possibles les mesures décisives pour son relèvement [...] enfin rien ne serait plus profitable à Michèle que l'humiliation dont elle allait être abreuvée 29 ». Sous couleur d'une rigueur logique, mise au service d'une prétendue miséricorde, s'enchaînent des constructions antithétiques qui recouvrent grossièrement un dessein inavouable : jouir du plaisir que procure une scène de délation. L'artifice de ce discours intérieur tient ainsi à la manipulation de la notion de finalité, à la substitution d'une finalité légitime à une finalité suspecte. Ces distorsions logiques révèlent le caractère spécieux, captieux d'un raisonnement qui présente quelque analogie avec la "mauvaise foi » sartrienne, dans la mesure où cette dissimulation à soi-même de ses motivations profondes est bien une manifestation d'inauthenticité ${ }^{30}$. Cette complaisance

28 III, p. 1366.

29 III, p. 767.

${ }^{30}$ Les êtres de mauvaise foi se réfèrent à des valeurs en soi auxquelles ils conforment aveuglément leur conduite. Ils se composent des personnages. Cette notion de "mauvaise foi " est théorisée et illustrée dans L'être et le néant (1943). Mais Sartre décrit et démasque la mauvaise foi dans des œuvres narratives qui précèdent la publication de La Pharisienne. Le négociant Pacôme de La Nausée (1938) se réfugie dans l'aveuglement de soi. Et, dans la dernière nouvelle de $L e$ Mur (1939), le jeune bourgeois Lucien Fleurier rejoint les camelots du roi, les rangs des antisémites, choisit la solution de l'inauthenticité et du confort moral. 
de la Pharisienne s'alimente à des arguties empruntées à un intertexte religieux.

La force de cette écriture romanesque tient au rayonnement sémantique induit par les phénomènes de stratification énonciative : d'une part, la superposition ou la juxtaposition des plans énonciatifs relatifs au narrateur et aux personnages, d'autre part l'hybridation des discours des personnages tissés de fragments hétérogènes, infiltrés par l'ironie.

Il n'est pas rare que le lecteur s'interroge sur la paternité des sources d'énonciation. La délimitation des aires énonciatives du narrateur et de l'héroïne est brouillée ou gommée par l'ambivalence des formes verbales (le jeu ménagé autour du conditionnel pour l'attribution de sa valeur modale ou temporelle), et par le glissement de certaines marques morphologiques du système du récit vers le système du discours (les pronoms de la troisième personne assument une fonction de déictique, de substitut d'un «je » propre au monologue rapporté). L'intervention subreptice du narrateur au sein du monologue narrativisé est assurée par de subtiles transitions. Ainsi, dans le chapitre XII, le cheminement des pensées de l'héroïne - en l'occurrence le processus d'autojustification -, est discrètement interrompu par le narrateur : "Il ne s'était rencontré personne pour lui apprendre qu'un homme, à mesure qu'il fraie sa route vers la sainteté, découvre un peu plus sa misère et son néant ${ }^{31}$ ». Lancée par une habile formule qui maintient provisoirement le discours indirect libre de pensée, la phrase nous introduit discrètement dans les vues du narrateur, qui ouvrent sur le paysage tragique de Pascal. La fusion des perspectives résulte également de l'emploi du présent gnomique qui, au sein de la clausule, entretient une ambiguiité, cisèle une conviction morale attribuable aussi bien au personnage qu'au narrateur, ou encore à l'auteur : " Au soir de sa vie, Brigitte Pian avait découvert enfin qu'il ne faut pas être semblable à un serviteur orgueilleux, soucieux d'éblouir le maître en lui payant son dû jusqu'à la dernière obole, et que Notre Père n'attend pas de nous que nous

31 III, p. 822. 
soyons les comptables minutieux de nos propres mérites. Elle savait maintenant que ce n'est pas de mériter qui importe mais d'aimer ${ }^{32}$ ». Le narrateur se pose en interprète de la parole évangélique pour fustiger la religion des certitudes et tirer un enseignement théologique des errements de la pharisienne.

De surcroît, l'intégration d'un autre discours dans la parole des personnages crée un effet polyphonique qui éveille chez le lecteur des résonances tantôt proprement mauriaciennes, tantôt religieuses. C'est ainsi l'intertexte mauriacien qui fonde la plurivocalité de ce récit. Une formule toute faite, martelée par la grand-mère de l'écrivain, « Tous les hommes sont des canailles », est attribuée à Brigitte Pian ${ }^{33}$. Maints aphorismes de l'abbé Calou portent en eux la parole du romancier. Comme celui qui envisage les retentissements spirituels des rencontres suggérés par la formule suivante, « les routes humaines ne se croisent pas au hasard ${ }^{34}$ » laquelle reprend, à la manière d'un écho, une idée-force de Mauriac consignée, par exemple, dans Destins (1928): «Les empreintes de l'homme sur l'homme sont éternelles et aucun destin n'a jamais traversé impunément le nôtre ${ }^{35}$ ".

Les semonces et les explications que l'abbé Calou destine à son élève, Jean de Mirbel, sont parcourues de citations non démarquées de l'Ancien et du Nouveau Testament. Telle phrase de l'abbé Calou - «Tu es de ceux que le Christ est venu chercher et sauver ${ }^{36} »-$, est partiellement empruntée à l'Évangile selon Saint-Luc ${ }^{37}$. L'intertexte évangélique façonne l'image de l'ange noir, de l'enfant réprouvé et racheté. Par ailleurs, les stéréotypes moralisants voisinent avec les citations bibliques dans les propos de Brigitte Pian. Si le réseau lexical de la direction de conscience

32 III, p. 881. L'édition de la Pléiade est entachée d'une erreur. L'adjectif "soucieux " a été omis dans le dernier paragraphe.

33 III, p. 873 et François Mauriac, Nouveaux mémoires intérieurs, Paris, Flammarion, 1965, p. 130.

34 III, p. 866.

35 II, p. 187.

36 III, p. 800.

${ }^{37}$ Evangile selon Saint-Luc, XIX, 10. 
(«brebis égarée, tentation, salut, miséricorde, mérites ») nourrit les reproches qu'elle destine à Octavie Tronche ou à Michèle Pian, des tournures telles que " touch[er] le fond de l'abîme » ou être "précipitée dans les ténèbres ${ }^{38}$ » ressortissent à la métaphorisation dévote de la faute charnelle. Identifiable par son empreinte livresque, la pensée verbalisée de la pharisienne est tissée de réminiscences du Nouveau Testament. L'épisode de la guérison de Saül par Jésus lui inspire l'image des " écailles [qui] tomberaient des yeux de M. Pian ${ }^{39}$ ». Ailleurs, un extrait de l'Épître aux Ephésiens de Saint Paul se présente sous la forme d'un énoncé interpolé dans le récit de pensée de Brigitte Pian. Il est détourné de sa signification originelle et mis au service de la glorification de soi ${ }^{40}$.

Propices au surgissement de l'ironie, les situations de coénonciation dans La Pharisienne s'appuient sur de subtiles combinaisons contrapuntiques. Ainsi trois voix se superposent ou se relaient dans une séquence du chapitre XII. Affectée d'une sourdine, la voix du narrateur laisse surgir les méditations de Brigitte Pian qui reprennent, à la manière d'un pastiche, les accents que la parabole évangélique prête au pharisien dans sa prière au temple ${ }^{41}$. Elle "remerci[ait] le Créateur qui l'avait faite créature si admirable 42 ». Élevant l'épithète laudative au superlatif implicite, l'autre voix s'insinue dans le discours intérieur du personnage, pour le subvertir à son insu, et faire apparaître l'imposture de l'examen de conscience. Le retournement ironique est opéré par l'intégration, dans le monologue narrativisé, d'une phraséologie religieuse chargée de mépris pour les êtres prisonniers du «bourbier» ou des «basses

38 III, p. 767.

${ }^{39}$ III, p. 767. Voir Actes des Apôtres, IX, 18. «Aussitôt tombèrent de ses yeux comme des écailles ».

${ }^{40}$ III, p. 821. «Ils ne voyaient pas comme elle sa perfection par le dedans: ils n'en mesuraient ni la hauteur, ni la largeur, ni la profondeur ». Ce fragment de monologue narrativisé reprend en le modifiant et en transformant sa signification un passage de l'Epître de Paul aux Ephésiens ( III, 18).

${ }^{41}$ Evangile selon Saint-Luc, (XVIII, 11).

42

III, p. 822. 
régions d'une ferveur suspecte ${ }^{43}$ ". Le discours à double entente a partie liée avec des images de labeur mesquin, affectées de caractérisations hyperboliques, et attribuées à la prétendue sainteté de la pharisienne. L'ironie souligne l'incohérence d'un personnage qui évolue dans un univers discursif régi par le mensonge ${ }^{44}$ et la bonne conscience. Le discours religieux est alors utilisé pour asseoir la stratégie de l'automystification, dont l'une des ramifications, la glorification de soi, se traduit par l'addition régulière d' « une maille à l'épais tissu de ses mérites ${ }^{45} »$. Le cliché du «tissu de mérite », remétaphorisé par l'image de la "maille», est complété par le qualificatif "épais », indice de la suffisance pharisienne, qui signale également la discordance entre la comptabilité minutieuse et l'amour véritable.

Dans La Pharisienne se manifeste donc avec éclat le talent d'un grand écrivain qui joue des ressources les plus diverses de l'écriture romanesque pour orchestrer les lignes vocales, pour saisir les couleurs, les modulations des voix et des représentations mentales, leur tressage comme leur superposition. Mauriac répond ainsi aux critiques sartriennes en montrant que son narrateur ne dispose pas des pouvoirs illimités traditionnellement impartis à Dieu. Mais l'écriture de la voix nous conduit à nuancer ce jugement. En effet, en dépit des limites imparties à sa situation, Louis Pian capte les pensées secrètes de sa belle-mère, observe de l'intérieur le champ d'une conscience pharisienne hantée par la comptabilité de ses mérites. Aussi arrive-t-il que les combinaisons polyphoniques soient menacées par le bruissement d'une monodie assignable à une voix omnisciente. Certes, c'est en usant

\section{${ }^{43}$ Ibid.}

44 Les variantes comportent une réflexion très critique de l'abbé Calou sur la fortune du mensonge au sein de la communauté chrétienne et sur les finalités stratégiques de son usage : "Mais à l'intérieur et comme à l'abri de la Vérité qu'ils détiennent, les chrétiens organisent le mensonge. [Le mensonge prolifère chez nous au centre même de la Vérité : les mensonges nécessaires à l'établissement temporel de l'église, à son organisation] », III, p. 1334. 
quelquefois des prérogatives d'un narrateur omniscient que le romancier appréhende l'envers du décor, mais cette liberté de manœuvre dans l'usage des techniques narratives lui a été reconnue par Sartre lui-même qui, en 1960, s'est exprimé dans ces termes à un journaliste de L'Express : «Je serai beaucoup moins vétilleux sur les méthodes. Toutes les techniques sont des trucages, y compris les techniques américaines ». Et notre plaisir de lecture ne tient-il pas à l'homologie qui se dessine entre les tentatives de la pharisienne pour se défaire de la direction de conscience et celles, tout aussi précaires du narrateur pour s'affranchir d'une perspective centralisatrice ? 